\title{
The fluctuating masker benefit for normal-hearing and hearing-impaired listeners with equal audibility at a fixed signal-to-noise ratio ${ }^{\text {a) }}$
}

\author{
Kenneth Kragh Jensen and Joshua G. W. Bernstein ${ }^{\text {b) }}$ \\ National Military Audiology and Speech Pathology Center, Walter Reed National Military Medical Center, \\ 4954 North Palmer Road, Bethesda, Maryland 20889, USA
}

(Received 19 October 2018; revised 9 March 2019; accepted 14 March 2019; published online 18 April 2019)

\begin{abstract}
Normal-hearing (NH) listeners can extract and integrate speech fragments from momentary dips in the level of a fluctuating masker, yielding a fluctuating-masker benefit (FMB) for speech understanding relative to a stationary-noise masker. Hearing-impaired (HI) listeners generally show less FMB, suggesting a dip-listening deficit attributable to suprathreshold spectral or temporal distortion. However, reduced FMB might instead result from different test signal-to-noise ratios (SNRs), reduced absolute audibility of otherwise unmasked speech segments, or age differences. This study examined the FMB for nine age-matched NH-HI listener pairs, while simultaneously equalizing audibility, SNR, and percentage-correct performance in stationary noise. Nonsense syllables were masked by stationary noise, $4-$ or $32-\mathrm{Hz}$ sinusoidally amplitude-modulated noise (SAMN), or an opposite-gender interfering talker. Stationary-noise performance was equalized by adjusting the response-set size. Audibility was equalized by removing stimulus components falling below the HI absolute threshold. HI listeners showed a clear 4.5-dB reduction in FMB for 32-Hz SAMN, a similar FMB to $\mathrm{NH}$ listeners for 4-Hz SAMN, and a non-significant trend toward a 2-dB reduction in FMB for an interfering talker. These results suggest that HI listeners do not exhibit a general diplistening deficit for all fluctuating maskers, but rather a specific temporal-resolution deficit affecting performance for high-rate modulated maskers. https://doi.org/10.1121/1.5096641
\end{abstract}

Pages: 2113-2125

\section{INTRODUCTION}

The human auditory system is remarkable in its ability to cope with other interfering sounds or speech when listening to target speech. When target speech is presented in a complex background of interference that modulates over time, the auditory system is able to take advantage of momentary dips in the level of masker to better hear the target speech, a process known as "dip listening" (Festen, 1993) or "glimpsing" (Cooke, 2006; Miller and Licklider, 1950). The resulting speech-intelligibility benefit provided for a fluctuating relative to a stationary masker has been termed "fluctuating-masker benefit" (FMB). Festen and Plomp (1990) found that hearing-impaired (HI) listeners receive dramatically less FMB than normal-hearing (NH) listeners when listening to speech masked by modulated noise or another interfering talker, a phenomenon that has been observed repeatedly since (e.g., Bernstein and Grant, 2009; Christiansen and Dau, 2012; Gustafsson and Arlinger, 1994; Hall et al., 2012; Lorenzi et al., 2006; Peters et al., 1998; Versfeld and Dreschler, 2002). It has been suggested that the reduced FMB for HI listeners reflects a deficit in the processing of supra-threshold information, such as a reduction in the

\footnotetext{
a) Portions of this work were previously presented at the jointly held 165th Meeting of the Acoustical Society of America, 21st International Congress on Acoustics, and 52nd Meeting of the Canadian Acoustical Association in Montreal, Canada, 7 June 2013 and the Midwinter Meeting of the Association for Research in Otolaryngology in Baltimore, Maryland, 11 February 2019.

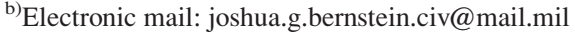

sharpness of peripheral tuning (e.g., ter Keurs et al., 1993), reduced temporal resolution (Dubno et al., 2003; George et al., 2006; Phatak and Grant, 2012), or a reduced ability to use temporal fine-structure information (Hopkins and Moore, 2009) to extract the target speech from the fluctuating background. There is reason to believe, however, that HI listeners might not face as much of a reduced ability to make use of supra-threshold processes to perform dip listening as many of these previous studies of FMB have suggested. There are at least two other important factors that could influence the FMB estimate for $\mathrm{HI}$ listeners that have not been systematically controlled: a difference in the SNR at which NH and HI listeners are tested (Bernstein and Grant, 2009) and a reduction in signal audibility that reduces the accessibility of target speech information during silent gaps in the masker level (Bacon et al., 1998; George et al., 2006).

Many studies of FMB for HI listeners could have been confounded by SNR differences between NH and HI listener groups (Bernstein and Grant, 2009; Freyman et al., 2012). The FMB is typically negatively correlated with SNR and thus larger at lower SNRs (Oxenham and Simonson, 2009), a phenomenon that has been attributed to an interaction between the distribution of speech information across the dynamic range and the distribution of instantaneous SNRs for a fluctuating masker (Bernstein, 2010; Bernstein and Grant, 2009). At low SNRs, most of the dynamic range of the target speech falls below the long-term root-meansquared (RMS) level of the masker, such that momentary dips in the fluctuating masker level will reveal a relatively 
large amount of speech information. At high SNRs, most of the dynamic range of the target speech will fall above the long-term RMS level of the masker, such that masker dips will reveal relatively little speech information. At the same time, momentary peaks in the masker level will tend to mask important portions of the target speech dynamic range, further reducing the FMB. Regardless of the cause, this SNR dependence means that the reduced FMB often observed for HI listeners might be at least partially attributable to the fact that they are generally tested at a higher stationary-noise SNR to yield an equal level of performance to NH listeners (Bernstein and Grant, 2009). When the FMB is evaluated at the same baseline $\mathrm{SNR}$ in $\mathrm{HI}$ and $\mathrm{NH}$ listeners, FMB differences between the two groups are substantially reduced. Equalizing the baseline SNR can be achieved in several different ways (for a review, see Bernstein, 2012), including estimating the FMB at different percentage-correct points for $\mathrm{NH}$ and $\mathrm{HI}$ listeners (Bernstein and Grant, 2009) or improving HI performance by testing auditory-visual conditions (Bernstein and Grant, 2009) or with a reduced-size closed-set of speech materials (Bernstein et al., 2012; Bernstein and Brungart, 2011).

Another important factor that could influence the FMB for HI listeners is the inaudibility of the target speech during dips in the level of the fluctuating masker due to elevated absolute thresholds. Elevated absolute thresholds are more likely to limit performance in the case of fluctuating maskers that contain many silent periods than for stationary noise where audibility of the speech signal is dominated more by the characteristics of the noise than the characteristics of the audiogram. Two different approaches have been used to limit the influence of differences in absolute threshold on the FMB. One approach has been to apply individualized (Phatak and Grant, 2012, 2014) or generic (Bernstein and Grant, 2009; George et al., 2006) frequency-specific gain in an attempt to increase signal audibility. The major limitation of this approach is that even if the mean speech level can be raised to be equal to or greater than absolute threshold for $\mathrm{HI}$ listeners, the dynamic range of speech is broad $(50 \mathrm{~dB}$; Zeng et al., 2002). This makes it nearly impossible to provide full audibility to the low-level portions of the speech signal without compression. Another consideration is that if $\mathrm{NH}$ and $\mathrm{HI}$ listeners are presented with different absolute signal levels, level effects on supra-threshold function could influence the results. In particular, the gain of the cochlear amplifier, thought to play an important role in providing temporal resolution in the auditory periphery (Nelson et al., 2001) is lower for higher input levels. The other approach has been to present NH listeners with an additional masking noise to simulate the elevated audiometric thresholds experienced by the HI listeners (Bacon et al., 1998; George et al., 2006). However, NH listeners may be able to listen further below the noise level to obtain speech information than the overall, averaged pure-tone threshold suggests (e.g., Drullman, 1995). Furthermore, modulation masking within auditory filters may occur for speech stimuli (Stone et al., 2012) but not for the detection of tones in noise that are used to estimate the audibility threshold. With both approaches (gain and masking noise), the measured deficit in FMB for HI relative to $\mathrm{NH}$ listeners has been shown to become smaller or non-existent. Yet, the limitations associated with these approaches suggests that audibility has not necessarily been equalized without causing other confounding effects.

The goal of the current study was to compare the FMB for $\mathrm{NH}$ and $\mathrm{HI}$ listeners while simultaneously controlling for audibility and SNR effects. Each HI listener was paired with a NH counterpart, age-matched to control for any possible effects of aging on the FMB (Dubno et al., 2003). To control for audibility differences, we presented identical signals to $\mathrm{NH}$ and HI listeners so as not to create any additional confounds related to differences in the stimuli. Instead of adding masking noise to the signal presented to NH listeners, which could have introduced modulation masking (Stone et al., 2012) that differed between the NH and HI listeners, we equalized audibility by employing a method of "intensity filtering" based on the ideal binary mask (Brown and Wang, 2005; Brungart and Iyer, 2012). The signal was divided into a grid of time-frequency elements, and only those elements whose level exceeded a given minimum threshold $(10 \mathrm{~dB}$ higher than the HI listener's audiometric threshold) were included in the recombined signal. Identical linear gain (as prescribed for the HI listener) was also applied to improve audibility, with low-pass filtering applied to avoid the excessive gain prescribed for frequencies above $4 \mathrm{kHz}$. To control for SNR differences, we employed the set-size adaptation method described by Bernstein et al. (2012). This speech task involved the identification of consonant-vowel (CV) or vowel-consonant (VC) tokens, with the size of the response set adaptively adjusted to yield a target percentage-correct performance level at a given stationary-noise SNR. Bernstein et al. (2012) demonstrated that this method could be used to adjust the overall difficulty of the task without affecting the FMB. Each NH-HI pair of listeners received the same target speech levels, the same SNRs, and the same audibility, with different set sizes adjusted to yield the same percentagecorrect level of performance in the stationary-noise condition. After this equalizing procedure, $\mathrm{NH}$ and $\mathrm{HI}$ performance was then compared in three different fluctuating-masker conditions [4- or 32-Hz sinusoidally amplitude modulated (SAM) noise or a single interfering talker] to determine whether the HI listeners would experience a reduced FMB.

\section{METHODS}

\section{A. Participants}

Eighteen listeners participated in the study: nine with $\mathrm{NH}$ and nine with sensorineural hearing loss. Each HI listener was paired with a NH counterpart matched as close in age as possible. Demographic and audiometric information for the test ear for the 18 listeners in the study are provided in Table I, while the average clinical audiograms for the test ear for the NH and HI study participants are shown in Fig. 1. The mean age for the HI listeners was 53.4 years (range 24-68 years) and the mean age for the NH listeners was 53.9 years (range 24-78 years), with an average absolute age difference between pairs of 2.0 years $( \pm 3.4 \mathrm{SD})$. There was a difference in the distribution of gender between the two groups: only two of the nine HI listeners were female, while seven out the nine NH listeners were female. Audiometric 
TABLE I. Demographic information for the nine HI and nine aged-matched NH subject participants, including clinical audiograms for the test ear.

\begin{tabular}{|c|c|c|c|c|c|c|c|c|c|c|c|}
\hline \multirow[b]{2}{*}{ Listener pair } & \multirow[b]{2}{*}{ Age (years) } & \multirow[b]{2}{*}{ Test ear } & \multirow[b]{2}{*}{ Sex } & \multicolumn{8}{|c|}{ Audiometric thresholds (dB HL) } \\
\hline & & & & 250 & 500 & 1000 & 2000 & 3000 & 4000 & 6000 & $8000 \mathrm{~Hz}$ \\
\hline \multicolumn{12}{|l|}{ HI listeners } \\
\hline 1 & 24 & $\mathrm{R}$ & M & 0 & 0 & 5 & 5 & 25 & 50 & 30 & 15 \\
\hline 2 & 47 & $\mathrm{R}$ & $\mathrm{F}$ & 35 & 45 & 60 & 60 & 55 & 55 & 65 & 60 \\
\hline 3 & 50 & $\mathrm{R}$ & M & 10 & 15 & 15 & 25 & 40 & 50 & 55 & 55 \\
\hline 4 & 63 & $\mathrm{R}$ & M & 40 & 45 & 50 & 50 & 50 & 55 & 60 & 50 \\
\hline 5 & 63 & $\mathrm{~L}$ & $\mathrm{~F}$ & 15 & 20 & 15 & 20 & 25 & 40 & 50 & 45 \\
\hline 6 & 68 & $\mathrm{R}$ & M & 35 & 40 & 50 & 60 & 65 & 60 & 65 & 60 \\
\hline 7 & 68 & $\mathrm{R}$ & M & 10 & 15 & 10 & 20 & 25 & 35 & 50 & 40 \\
\hline 8 & 37 & $\mathrm{~L}$ & M & 0 & 15 & 50 & 50 & 55 & 55 & 35 & 25 \\
\hline 9 & 61 & $\mathrm{R}$ & M & 5 & 10 & 15 & 40 & 45 & 55 & 70 & 75 \\
\hline \multicolumn{12}{|l|}{ NH listeners } \\
\hline 1 & 24 & $\mathrm{R}$ & $\mathrm{F}$ & 0 & 5 & 5 & 5 & 0 & 0 & 5 & 5 \\
\hline 2 & 47 & $\mathrm{R}$ & $\mathrm{F}$ & 10 & 5 & 15 & 10 & 15 & 5 & 5 & 10 \\
\hline 3 & 50 & $\mathrm{~L}$ & $\mathrm{M}$ & 5 & 5 & 5 & 20 & 5 & 15 & 10 & 0 \\
\hline 4 & 60 & $\mathrm{~L}$ & $\mathrm{~F}$ & 20 & 15 & 5 & 10 & 20 & 20 & 15 & 25 \\
\hline 5 & 59 & $\mathrm{~L}$ & $\mathrm{~F}$ & 5 & 10 & 10 & 5 & 20 & 15 & 35 & 30 \\
\hline 6 & 69 & $\mathrm{R}$ & $\mathrm{F}$ & 5 & 5 & 5 & 15 & 35 & 5 & 5 & 20 \\
\hline 7 & 78 & $\mathrm{R}$ & $\mathrm{F}$ & 20 & 10 & 20 & 25 & 25 & 15 & 20 & 25 \\
\hline 8 & 37 & $\mathrm{~L}$ & M & 5 & 0 & 5 & 5 & 10 & 10 & 15 & 10 \\
\hline 9 & 61 & $\mathrm{~L}$ & $\mathrm{~F}$ & 5 & 10 & 5 & 10 & 10 & 15 & 25 & 5 \\
\hline
\end{tabular}

inclusion criteria were based on clinical audiogram data for octave frequencies between 250 and $4000 \mathrm{~Hz}$. The NH listeners had pure-tone thresholds no greater than $25 \mathrm{~dB}$ hearing level (HL). The HI listeners had sensorineural hearing loss in both ears, with air conduction thresholds of at least $35 \mathrm{~dB} \mathrm{HL}$ for at least one frequency, and no air-bone gap larger than $10 \mathrm{~dB}$. Three of the HI listeners had audiogram asymmetries greater than $15 \mathrm{~dB}$ for at least one frequency and were tested in their better ear. None of the other $\mathrm{NH}$ or $\mathrm{HI}$ listeners had asymmetries greater than $15 \mathrm{~dB}$; these listeners were tested in the ear of their choice. After the experiment was completed it was observed that two of the $\mathrm{NH}$ listeners had audiometric thresholds in the test ear that exceeded $25 \mathrm{~dB} \mathrm{HL}$ at audiometric frequencies that were not specified in the inclusion criteria (NH listener 5 at 6000 and $8000 \mathrm{~Hz}$; NH listener 6 at $3000 \mathrm{~Hz}$ ). To examine whether the

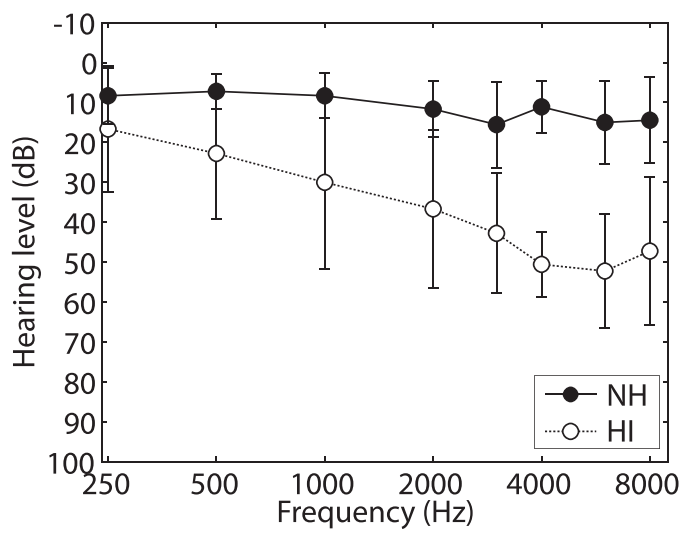

FIG. 1. Mean clinical audiograms (hearing level as a function of frequency) for the NH and HI listeners. Error bars indicate \pm 1 standard deviation. $\mathrm{HI}=$ hearing-impaired; $\mathrm{NH}=$ normal-hearing. inclusion of these two notionally NH listeners affected the experimental results, all statistical analyses were repeated while excluding these two NH listeners and their paired $\mathrm{HI}$ counterparts. All listeners reported English as their first language, and that they had no history of learning disabilities, stroke, disabling mental illness, severe head injury, or neurological disorder. The study was approved by the Institutional Review Board of Walter Reed National Military Medical Center and all listeners provided informed consent.

\section{B. Materials}

\section{Target speech}

The speech stimuli consisted of a set of 160 nonsense $\mathrm{CV} / \mathrm{VC}$ syllables spoken by seven different male talkers (Bernstein et al., 2012) taken from the Linguistic Consortium LDC-2005S22 corpus (Fousek et al., 2004) and presented at a fixed level of $80 \mathrm{~dB}$ sound pressure level (SPL). The set consisted of all CV/VC combinations of the following five different vowels " $a h$ " as in rod, "ay" as in raid, "ee" as in reed, "oh" as in road, and "oo" as in rude and the following 16 consonants " $b$, , " ch," " $d, "$ " $f$, , " $g$, , " j,,"

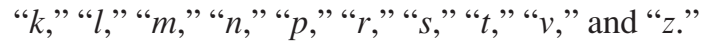

\section{Maskers}

Psychometric functions were measured for four masker types identical to those used in Bernstein et al. (2012): (1) a stationary, speech-shaped noise based on an average of the 160 tokens spoken by each of the seven talkers (a total of 1120 tokens); (2) a 4-Hz SAM version of the stationary speech-shaped noise, with $100 \%$ modulation depth; (3) a 32$\mathrm{Hz}$ SAM noise; and (4) a female interfering talker (mean $F 0=199 \mathrm{~Hz}$ ). The interfering talker was a native speaker of 
American English and read passages from the "Unfruitful Tree" by Freidrich Adolph Krummacher (translated from German). The interfering speech was filtered to have a longterm average spectrum equal to that of the stationary and SAM noises. For all masker types, the masker was ramped on $300 \mathrm{~ms}$ before the target speech and ramped off $300 \mathrm{~ms}$ after it. The response set was presented on a grid of software response buttons arranged with the vowels in the rows and the consonants in the columns. The response panel had a separate upper grid for the $\mathrm{CV}$ combinations and a lower grid with the VC combinations.

\section{Equalization of audibility}

The current study sought to control audibility differences between $\mathrm{HI}$ and $\mathrm{NH}$ listeners while avoiding the complications associated with the use of noise masking, different levels, or different gain for the two listener groups. This was done by the use of intensity filtering to filter spectrotemporal elements below the hearing threshold of the HI listener. To be able to do the intensity filtering as accurately as possible, the $\mathrm{HI}$ absolute threshold was determined using the same apparatus (sound card, headphones, and calibration) as the speech-perception testing.

\section{Measurement of absolute thresholds}

Absolute thresholds for the HI listeners were measured using a three-alternative forced-choice paradigm. Two intervals contained only silence, while the third interval contained a $150-\mathrm{ms}$ pure-tone with 10 -ms raised-cosine onset and offset ramps. The three intervals were indicated by a change in the color of the appropriate response button. A two-down, one-up paradigm was used to track the absolute tone level required for $70.7 \%$ correct performance in identifying the interval containing the tone stimulus (Levitt, 1971). Two measurements were repeated for each of the following test frequencies presented in random order: 125, 250, $500,1000,1500,2000,3000,4000$, and $5000 \mathrm{~Hz}$. The absolute threshold was taken to be the average of the two measurements.

\section{Frequency filtering and $\mathrm{HI}$ gain prescription}

Prior to the intensity filtering, the speech stimuli and noise were low-pass filtered at $4 \mathrm{kHz}$ (24th-order Butterworth) to avoid the requirement to apply large gain to compensate for the typically large hearing losses at higher frequencies. Next the spectrum was optimally shaped for each individual HI listener by applying the Cambridge formula (Moore and Glasberg, 1998) based on the listener's absolute threshold measured in the setup as specified above. The exact same stimulus processing (low-pass filtering and frequency-dependent linear gain) was also applied for each HI listener's NH counterpart. Finally, the overall level was set to $80 \mathrm{~dB}$ SPL. Thus, each matched pair was presented with identically processed stimuli.

\section{Intensity filtering}

Intensity filtering was applied to remove any speech information remaining inaudible to the HI listener after the applied gain and low-pass filtering. This processing removed any speech (or masker) elements that fell below a criterion level of $10 \mathrm{~dB}$ above the absolute threshold of the $\mathrm{HI}$ listener (see above). The 10-dB criterion was chosen instead of $0 \mathrm{~dB}$ as a safety margin to better assure equal audibility. The intensity filtering was based on the ideal binary mask method (Brown and Wang, 2005; Brungart and Iyer, 2012) where the signal was first gammatone filtered into 32 frequency bands and then each band sectioned into 20-ms time bins (Fig. 2). A binary mask was created consisting of a matrix with an entry for each of the frequency-time bins. Any frequency-time segment of the signal that had energy lower than $10 \mathrm{~dB}$ above absolute threshold of the HI listener received a " 0 " entry in the matrix, and a " 1 " otherwise. An intensity-filtered signal was then re-synthesized where all signal segments with a " 0 " entry were removed and segments with a " 1 " entry were added into the synthesis mix with a cosine window applied. The masker was processed separately, but identically to the target speech, before being combined with the processed target speech. This was done to ensure that no speech information falling below the HI threshold of audibility would be passed on in time-frequency bins where the masker level was dominant, and therefore be potentially audible to the $\mathrm{NH}$ listener even at a negative SNR. The gains of the gammatone filters employed in the algorithm were calibrated to ensure that the energy passed equally in all frequency bands and was thus intensity filtered equally. This was done by passing a $150-\mathrm{ms}$ pure tone at the center of each frequency band to producing a transfer function that was applied for energy equalization after gammatone filtering.

To validate the intensity-filtering algorithm, pilot tests were carried out for two $\mathrm{NH}$ and two HI listeners to ensure that the processing produced the desired detection thresholds. The audiogram measurement was repeated using pure tones that were processed with the algorithm. This procedure produced a binary result - tones presented at a level below the intensity-filtering criterion were completely inaudible, while tones presented above the criterion level were detected $100 \%$ of the time.

\section{Equipment}

The participants were seated in a sound-proof room in front of a touchscreen panel operating a GUI interface with a total of 160 software buttons, each with one of the nonsense syllables spelled out (see above). The touch panel was connected to the experimental PC running an automated custom MATLAB procedure. Participants wore calibrated HD280 pro closed circumaural headphones (Sennheiser, Wedemark, Germany) connected to a Hammerfall DSP Multiface II sound card (RME Audio, Haimhausen, Germany).

\section{E. Procedure}

For each matched listener pair, the HI listener was tested first, because of the requirement to obtain $\mathrm{HI}$ audiometric 
(A)

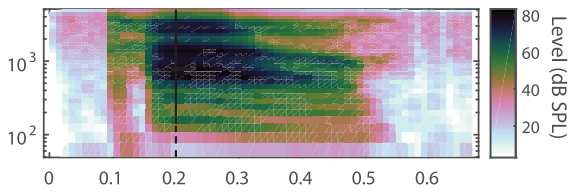

(B)

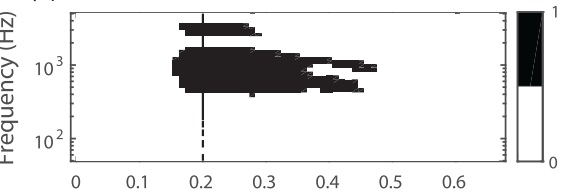

(C)

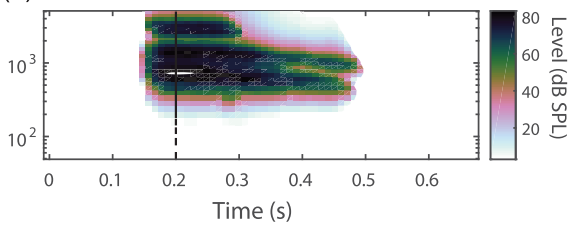

(D)

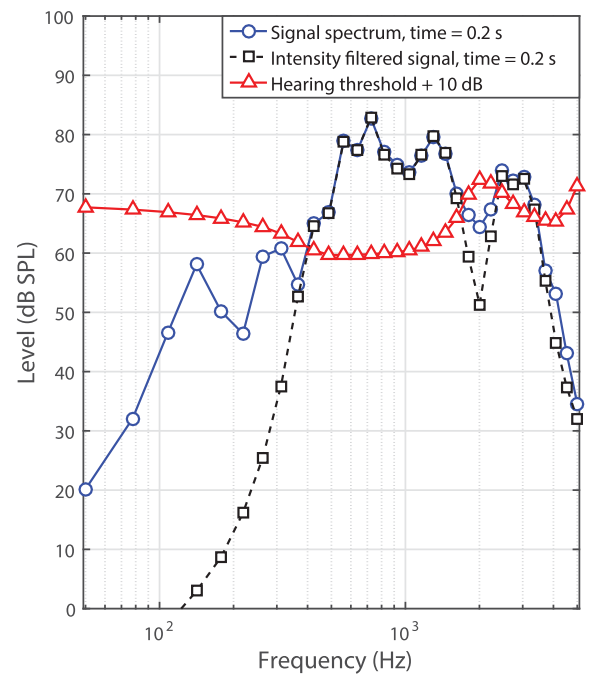

FIG. 2. (Color online) Illustration of the intensity-filtering algorithm. (a) Spectrogram of speech nonsense syllable "poh" after low-pass filtering at $4 \mathrm{kHz}$ and applying linear gain according to the Cambridge formula. (b) Binary mask consisting of 32 frequency bands and time segments of 20 -ms duration each. The total energy was calculated for each time-frequency segment. Segments where the energy exceeded the given hearing-impaired absolute threshold plus 10 dB were assigned the value of one (black) and segments below this level were assigned the value of zero (white). (c) A new signal containing only suprathreshold time-frequency segments was then synthesized by multiplying the binary mask (b) and original signal (a). (d) The stimulus spectrum for the 20-ms speech segment at time $0.2 \mathrm{~s}$ [dotted lines in (a)-(c)], represented by the energy within each gammatone filter (blue circles), plotted together with the intensityfiltering criterion (absolute threshold $+10 \mathrm{~dB}$; red triangles) and the resulting intensity-filtered signal (black asterisks). SPL $=$ sound-pressure level.

information to inform the signal processing for both listeners in the pair. The test procedure for each HI listener consisted of three steps. The first step consisted of absolute threshold measurements made using the same laboratory equipment and calibration that were used for the speech testing (see Sec. II C 1, above). These measurements ensured the accuracy of the threshold measurement with respect to the intensity-filtering process. The second step determined the set size required to yield a fixed level of syllableidentification performance $(66.7 \%$ correct $)$ in stationary speech-shaped noise at one SNR $(-3 \mathrm{~dB})$ using adaptive tracking. The third step was the main experiment, whereby a method of constant stimuli was used to measure syllableidentification performance for all eight SNRs for each of the four masker types tested. NH listeners completed only the second step (set-size determination) and third step (main experiment) since only the HI absolute threshold was needed to determine the signal processing to be imposed for each matched NH listener.

\section{Preliminary experiment: Set-size determination}

To eliminate any potential remaining differences in stationary-noise performance between the NH and HI listeners after equalization of audibility, a preliminary experiment employed the method developed in Bernstein et al. (2012) to estimate the set size required for a desired level of performance for each listener. To render the desired set size on any given trial, only a subset of all 160 responses were made available. A given subset was selected from the full set in a pseudo-random manner such that the subset was distributed as equally as possible across $\mathrm{VC}$ and $\mathrm{CV}$ groups and the available vowels and consonants. Listeners identified the target $\mathrm{CV}$ or $\mathrm{VC}$ nonsense syllable by selecting one of the activated buttons. Feedback was provided following each trial by highlighting the correct response.

Target speech was presented in stationary speech-shaped noise at an SNR of $-3 \mathrm{~dB}$, with the SNR defined before the application of the intensity-filtering algorithm to the target speech and maskers. The initial set size was set to 8 . The set size was multiplied by a factor of $2^{1 / 4}$ following any two correct responses, and divided by a factor of $2^{1 / 4}$ following any incorrect response, thereby tracking the set-size required for $66.7 \%$ correct performance (similar to the 75\%, 3-up, 1-down procedure described by Zwislocki and Relkin, 2001). When the tracked set size was a non-integer value, the actual set size on a given trial was determined by randomly rounding the tracked set size up or down in proportion to the decimal portion of the tracked set size. For example, for a tracked set size of 10.25 , the actual set size was set at 10 with probability 0.75 and 11 with probability 0.25 , yielding an average actual set size of 10.25 . The set-size estimation procedure was run for a total of 200 trials. The first 160 trials served as training, and the final set size for a given listener was taken to be the geometric average of set sizes on the last 40 trials.

\section{Main experiment: Measurement of psychometric functions for all masker types}

After the appropriate set size was determined for a given listener (see above), the main experiment was carried out to evaluate performance as a function of SNR for each masker type. The set size was held fixed for each listener at the value estimated in the preliminary experiment, and psychometric functions were measured at eight different SNRs for each of the four masker types. For stationary noise, the range of test SNRs was -9 to $12 \mathrm{~dB}$ in 3-dB steps. For the three fluctuating maskers, the range of test SNRs was -12 to $9 \mathrm{~dB}$ in $3-\mathrm{dB}$ steps. The pseudo- 

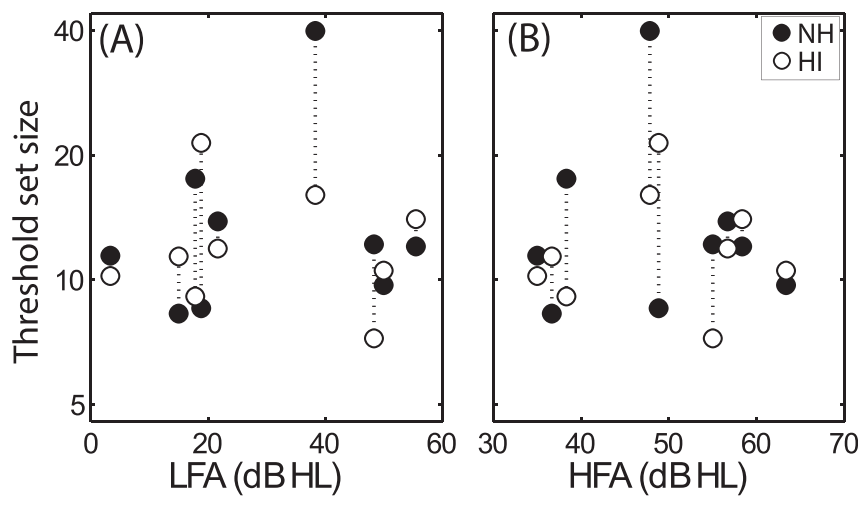

FIG. 3. Results of the preliminary experiment plotting the threshold set size-defined as the set size required for $66.7 \%$ correct speech-identification performance in stationary noise at a signal-to-noise ratio of $-3 \mathrm{~dB}$-for each individual listener as a function of (a) the LFA (500, 1000, and $2000 \mathrm{~Hz}$ ) and (b) the HFA audiometric threshold $(3000,4000$, and $6000 \mathrm{~Hz})$ for the HI listener in each matched pair. Vertical dashed lines connect the data points for each $\mathrm{NH}-\mathrm{HI}$ listener pair. HFA = high-frequency average; $\mathrm{HI}=$ hearing-impaired; $\mathrm{HL}=$ hearing level; LFA $=$ low-frequency average; $\mathrm{NH}=$ normal-hearing.

random selection of active buttons changed on each trial. Stimuli were presented in blocks of 26 trials. In each block, the masker type was held constant, and 13 trials were presented for each of two test SNRs selected at random from the eight available choices. Two blocks were repeated for each combination of masker type and SNR pair, for a total of 26 trials for each SNR and masker type (832 trials total) for each listener.

\section{RESULTS}

\section{A. Preliminary experiment: Equalization of performance in stationary noise}

The threshold set size was defined as the set size required to yield $66.7 \%$ correct performance at an SNR of $-3 \mathrm{~dB}$ for each individual listener. The geometric-mean threshold set size was 12.6 syllables for the NH listeners and 12.2 syllables for the HI listeners. A paired t-test conducted on the log-transformed data indicated that the threshold set size was not significantly different between the $\mathrm{NH}$ and $\mathrm{HI}$ listeners $[\mathrm{t}(8)=0.15, \mathrm{p}=0.89]$. Figure 3 plots the threshold set sizes for each listener pair as a function of the HI lowfrequency average (LFA) audiometric threshold [500, 1000, and $2000 \mathrm{~Hz}$; Fig. 3(A)] and high-frequency average (HFA) threshold [3000, 4000, and $6000 \mathrm{~Hz}$; Fig. 3(B)]. The vertical dotted lines in Fig. 3 connect each matched NH (filled circles) and HI (open circles) listener pair. No significant correlations were observed between the LFA or HFA and the threshold set size, nor were any significant correlations observed between the LFA or HFA and the effect of hearing loss on threshold set size (i.e., the length of the dotted lines).

\section{B. Main experiment: Psychometric functions for stationary-noise and fluctuating-masker conditions and the FMB}

Figure 4 shows the results of the main experiment. Each panel shows the results for one of the masker conditions. The black symbols represent mean performance $( \pm 1$ standard error) for the NH listeners, while the white symbols represent mean performance for the HI listeners. For each listener group and condition, the data were fit with a sigmoidal function with two free parameters describing the slope and intercept of the function. In addition to these two parameters for each group and condition, the fitting process also identified a single maximum performance plateau for each sigmoidal function of $88.1 \%$ correct, held fixed across all test conditions and both listener groups, that allowed the best global fit to the entire data set. The fitted sigmoidal functions were also assumed to have a minimum plateau value of $0 \%$ correct. The solid curves represent sigmoidal fits for the $\mathrm{NH}$ listeners and the dotted curves represent sigmoidal fits for

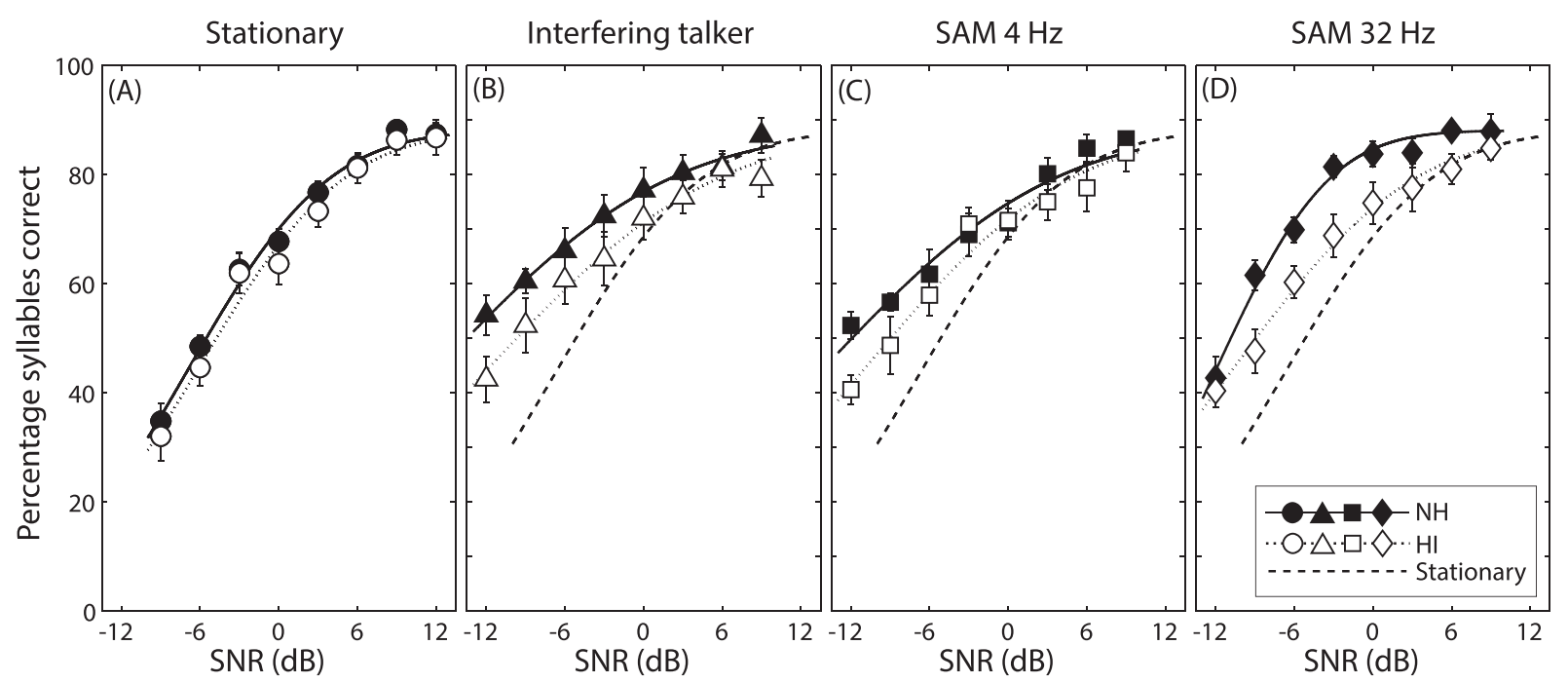

FIG. 4. Results of the main experiment. The percentage of syllables correctly identified is plotted as a function of SNR along with fitted psychometric functions for the NH (black symbols and solid curves) and HI listeners (white symbols and dotted curves). Results for each masker type are plotted in separate panels: (a) stationary noise, (b) interfering talker, (c) 4-Hz SAM noise, and (d) 32-Hz SAM noise. The fitted curve for (a) the stationary-noise condition (dashed curve, same for NH and HI listeners) is replotted in panels (b)-(d) for comparison with each fluctuating-masker condition. Error bars indicate \pm 1 standard error. $\mathrm{HI}=$ hearing-impaired; $\mathrm{NH}=$ normal-hearing; $\mathrm{SAM}=$ sinusoidally amplitude-modulated; $\mathrm{SNR}=$ signal-to-noise ratio. 
the HI listeners. Because stationary-noise performance was not significantly different for the NH and HI listeners [Fig. 4(A)], the average of the fitted curves for the stationarynoise condition is represented in each of the other panels as a single dashed curve for comparison with the fluctuatingmasker data. Figure 5 plots the FMB for each test SNR that was common to the stationary and fluctuating-masker conditions. The FMB, expressed in percentage points of improvement, was derived from the raw data in Fig. 4 by calculating the percentage-point difference in performance between the stationary-noise condition and each fluctuating-masker condition.

The first question to examine was whether the set-size manipulation successfully equalized stationary-noise performance between the NH and $\mathrm{HI}$ listeners. Stationary-noise performance was compared for the $\mathrm{NH}$ and $\mathrm{HI}$ listeners using a repeated-measures logistic regression analysis (RMLRA) that treated SNR, hearing status (NH or HI), and listener pair (1-9) as fixed effects, and individual listener as a random effect. Treating listener pair as a fixed effect allowed the model to capture the fact that each matched pair of listeners was presented with the same intensity-filtered stimuli, but that the filtering parameters varied across the different listener pairs. The model accounted for this paired design by subtracting out the average performance for each listener pair, thereby focusing on the difference in performance between each paired NH and HI listener. Treating the individual listener as a random effect captured any random variability associated with each of the 18 individual participants. There were significant main effects of SNR $\left[\chi^{2}(7)=1090\right.$, $\mathrm{p}<0.0001]$ and listener pair $\left[\chi^{2}(8)=43.9, \mathrm{p}<0.0001\right]$, but no significant effect of hearing status $(\mathrm{p}=0.11)$ or interactions between hearing status and SNR $(p=0.98)$. After establishing that the $\mathrm{NH}$ and $\mathrm{HI}$ listeners had equal stationary-noise performance, the next question was whether the magnitude of the FMB differed between the $\mathrm{NH}$ and $\mathrm{HI}$ listeners for any of the fluctuating-masker conditions. The FMB was characterized after transforming the raw performance data (Fig. 4) into rau units to stabilize the variance (Studebaker, 1985). The FMB data were then analyzed using a RM-LRA that treated SNR, masker type, and hearing status, and listener pair as fixed effects, and listener as a random effect. The main effects of SNR, masker type, and hearing status were included in the model together with all of their interactions. To limit the total number of terms in the model, only the main effect of listener pair was included in the model without any associated interaction terms.

There were significant main effects of hearing status $\left[\chi^{2}(1)=13.6, \mathrm{p}=0.0002\right], \mathrm{SNR}\left[\chi^{2}(6)=194, \mathrm{p}<0.0001\right]$, masker type $\left[\chi^{2}(2)=20.4, \mathrm{p}<0.0001\right]$, and listener pair $\left[\chi^{2}(8)=237, \mathrm{p}<0.0001\right]$. There was a significant two-way interaction between SNR and masker type $\left[\chi^{2}(12)=78.9\right.$, $\mathrm{p}<0.0001]$, reflecting the observation that the effect of SNR on the FMB differed depending on the masker type. Most importantly for the main question posed in the study (i.e., the effect of hearing loss on the FMB) there was a significant two-way interaction between hearing status and masker type $\left[\chi^{2}(2)=8.60, \mathrm{p}=0.014\right]$ and a significant three-way interaction between hearing status, masker type and SNR $\left[\chi^{2}(12)=36.2, p<0.0003\right]$. These interactions indicate that the effect of hearing loss on the FMB varied depending on masker type and SNR. The interaction between hearing status and SNR was not significant $(\mathrm{p}=0.90)$.

Post hoc pairwise comparisons examined the interactions involving hearing status and masker type. In the comparisons between the FMB for each of the three masker types averaged across SNR, Bonferroni corrections for multiple (three) comparisons were applied by reducing the criterion $\mathrm{p}$-value (i.e., $\mathrm{p}<0.0167$ ). There was a significant difference between the $\mathrm{NH}$ and $\mathrm{HI}$ FMB for the 32-Hz SAM noise masker $\left[\chi^{2}(1)=16.5, \mathrm{p}<0.0001\right]$, whereby the HI listeners obtained an average of 7 percentage points (range

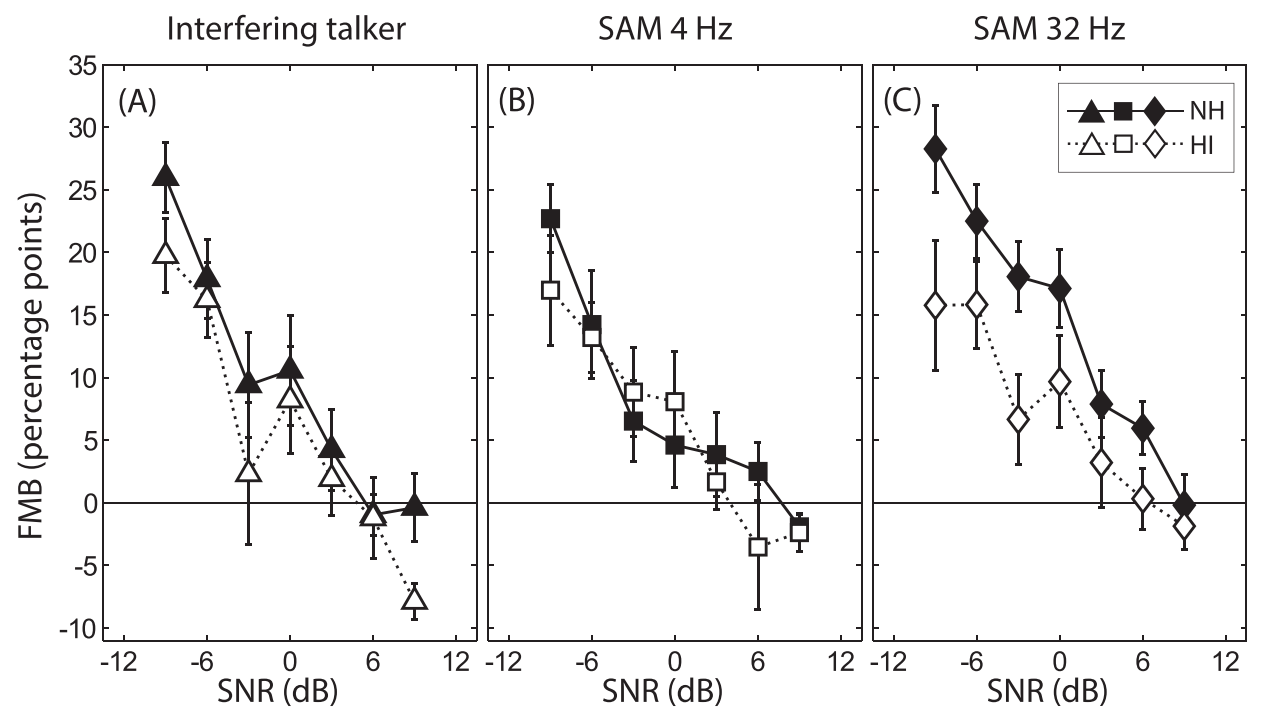

FIG. 5. The FMB in percentage points is plotted as a function of SNR for the NH (black symbols and solid curves) and HI listeners (white symbols and dotted curves). The FMB was derived from the data plotted in Fig. 4 as the difference in percentage-correct syllable-identification performance between each fluctuating-masker condition and the stationary-noise masker condition. Each panel represents a different fluctuating-masker type: (a) interfering talker, (b) 4$\mathrm{Hz}$ SAM noise, and (c) 32-Hz SAM noise. FMB = fluctuating-masker benefit; $\mathrm{HI}=$ hearing-impaired; $\mathrm{NH}=$ normal-hearing; SAM = sinusoidally amplitudemodulated; $\mathrm{SNR}=$ signal-to-noise ratio. 

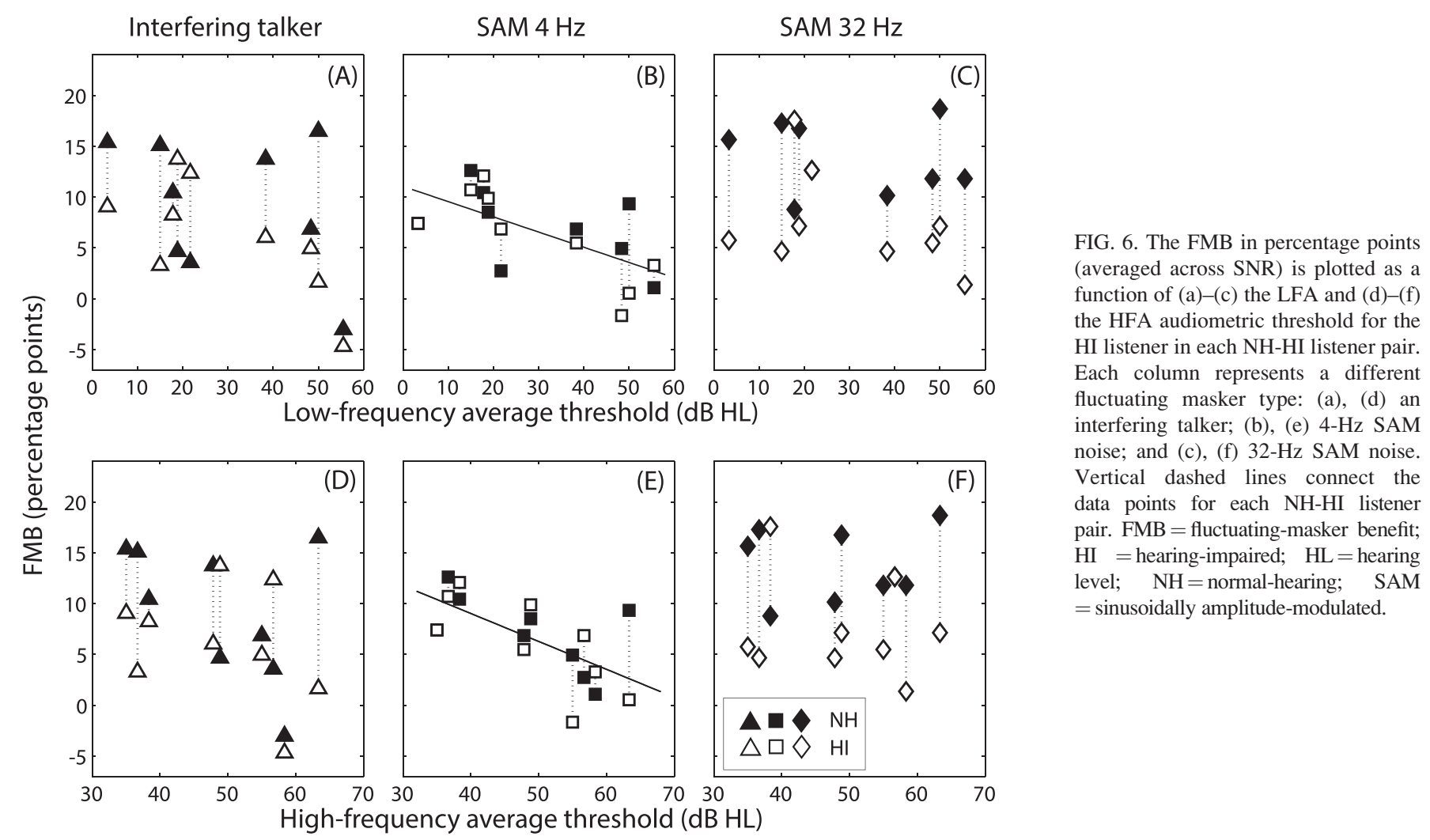

$=$ sinusoidally amplitude-modulated.

2\%-13\%) less FMB than the NH listeners [Fig. 5(C)]. This difference can also be expressed in decibel terms by calculating the horizontal distance between the fitted functions in Fig. 4(D): the HI listeners obtained an average of $4.5 \mathrm{~dB}$ (range: $0-9 \mathrm{~dB}$ ) less FMB than the NH listeners for the 32$\mathrm{Hz}$ SAM noise masker. No significant differences were observed between the NH and HI FMB for the 4-Hz SAM noise maskers $\left[\chi^{2}(1)=1.05, p=0.31\right]$. For the interferingtalker masker, the trend toward a slightly reduced FMB for the $\mathrm{HI}$ listeners ( 4 percentage points or $2 \mathrm{~dB}$ less than the $\mathrm{NH}$ listeners) was not found to be significant $\left[\chi^{2}(1)=3.23\right.$, $\mathrm{p}=0.072 \mathrm{]}$. Pairwise comparisons examining the effect of hearing status on the FMB were also carried out for each combination of SNR and masker type, with Bonferroni corrections for (seven) multiple comparisons (criterion p-value $0.05 / 7=0.0071)$. The difference between the $\mathrm{NH}$ and $\mathrm{HI}$ FMB was not found to be significantly different at any SNR for any masker type. In summary, a significant difference in FMB between the NH and HI listeners was observed for 32$\mathrm{Hz}$ SAM noise when the data were pooled across SNR, but was not observed in the post hoc comparisons at each individual SNR.

To examine whether the inclusion of the two notionally $\mathrm{NH}$ listeners with mild threshold elevation affected the results, the statistical analyses were repeated while excluding these two NH listeners and their matched HI counterparts (i.e., listener pairs 5 and 6 ). Although there were some small changes in the resulting $\chi^{2}$ and p-values, the pattern of statistically significant main effects, interactions, and post hoc comparisons was nearly identical to the full analysis that included all nine listener pairs. The only exception was that in the post hoc analysis for the seven listener pairs, the difference in FMB between the NH and HI listeners became significant for the 32-Hz SAM noise masker at an SNR of $0 \mathrm{~dB}(\mathrm{p}=0.0033)$.

One possible reason for the lack of significant differences in FMB for the 4-Hz SAM noise and interfering-talker maskers is that the sample of HI participants included a number of listeners with normal or near-normal thresholds at frequencies of $2 \mathrm{kHz}$ and below (HI listeners 1, 3, 5, and 7; Table I). To examine whether the degree of hearing loss influenced the magnitude of the FMB, Fig. 6 plots the mean FMB (averaged across SNR) for each NH and HI listener as a function of the LFA (top row) or HFA threshold (bottom row) for the HI listener in each pair. The horizontal axes in Fig. 6 can be interpreted in terms of the effect of audibility on performance, given that the signals were processed to ensure that speech information below the HI threshold was inaudible for both listeners in each matched pair. The vertical dotted lines in Fig. 6 connect each NH-HI listener pair, with the length of the dotted line representing the effect of hearing loss on the FMB. Any FMB difference between the $\mathrm{NH}$ and $\mathrm{HI}$ listener in each pair can be interpreted as an effect of suprathreshold distortion, given that audibility was equalized for each pair.

For each panel in Fig. 6, the FMB data were analyzed with a linear regression model that included hearing status as an independent variable and LFA or HFA as a covariate. Applying Bonferroni corrections for six comparisons, the significance criterion was set to $\mathrm{p}<0.083$ (i.e., 0.05/6). For the 4-Hz SAM noise masker [Figs. 6(B) and 6(E)], there was a clear effect of audibility on the magnitude of the FMB, with significant effects of the LFA $\left[\chi^{2}(1)=13.6, p=0.0002\right]$ and the HFA $\left[\chi^{2}(1)=15.1, \mathrm{p}=0.0001\right.$ ] [diagonal lines in 
Figs. 6(B) and 6(E) represent a linear fit to the FMB data across all 18 individual listeners]. There was, however, no evidence of an effect of hearing loss on the FMB in either model $(p=0.45-0.46)$, consistent with lack of effect observed in the group data [Fig. 5(B)]. For the 32-Hz SAM noise masker [Figs. 6(C) and 6(F)], the converse was true. There was no evidence of a relationship between the LFA $(p=0.18)$ or HFA $(p=0.68)$ and the FMB, but a significant effect of hearing loss $(p=0.0003-0.0007)$, consistent with the group data [Fig. 5(A)]. For the interfering-talker masker [Figs. 6(A) and 6(D)], there were a non-significant trend toward an effect of LFA $\left[\chi^{2}(1)=6.11, p=0.013\right]$ on the FMB, but no evidence of an effect of hearing loss in either model $(p=0.21-0.45)$. A second analysis found no significant correlations between the LFA or HFA and the magnitude of the effect of hearing loss on the FMB (i.e., the lengths of the vertical lines in Fig. 6) for any of the three maskers $(p=0.20-0.76)$. Thus, there was no evidence of a reduced FMB for the 4- $\mathrm{Hz}$ SAM noise or interfering talker, even for the HI listeners with the most elevated audiometric thresholds.

\section{DISCUSSION}

\section{A. Summary of main findings}

The goal of this study was to examine the FMB for masked speech perception for age-matched NH and HI listeners presented with the same signals, audibility, and level of performance in the baseline stationary-noise conditions. Equal audibility was accomplished using a combination of frequency-dependent gain, low-pass filtering, and intensity filtering based on the concept of an ideal binary mask, while equal performance in stationary noise was achieved by adjusting the size of the closed response set. These manipulations successfully achieved the goal of equalizing stationary-noise performance for the $\mathrm{NH}$ and $\mathrm{HI}$ listeners. The HI listeners showed a clear $4.5-\mathrm{dB}$ reduction in the magnitude of the FMB relative to the NH listeners for $32-\mathrm{Hz}$ SAM noise. There was no observed effect of hearing loss on the FMB for the 4-Hz SAM noise masker or for the interfering talker, although in the interfering-talker case there was a non-significant trend for the FMB to be reduced for the HI listeners by about $2 \mathrm{~dB}$ relative to the $\mathrm{NH}$ listeners.

\section{B. Comparison with previous studies}

These results contrast with many previous studies that have compared the FMB for NH and HI listeners. These studies have generally found that hearing impairment reduces the FMB for a wide variety of masker types, with the magnitude of the reduction greater than what was observed here. For example, a number of studies that did not control for audibility or SNR effects identified a reduction in the FMB experienced by HI listeners of 4-7 dB (Festen and Plomp, 1990; Peters et al., 1998; Versfeld and Dreschler, 2002) or 15-25 percentage points (Gustafsson and Arlinger, 1994; Lorenzi et al., 2006). Some previous studies that controlled for SNR differences but not for audibility differences also identified a reduction in the FMB for $\mathrm{HI}$ listeners on the order of 1-5 dB (Bernstein and Grant, 2009; Christiansen and Dau, 2012; Hall et al., 2012). Other previous studies attempted to control for audibility effects by applying masking noise to the signals presented to $\mathrm{NH}$ listeners or by applying individualized gain to the signals presented to the HI listeners, but did not explicitly control SNR effects. In these cases, HI listeners showed a reduction in FMB of 2-7 dB (Eisenberg et al., 1995; George et al., 2006). Thus, many previous studies of FMB in the literature found a reduced FMB for HI listeners, but these studies did not control both SNR and audibility effects.

There are a handful of previous studies of FMB that, like the current study, controlled for audibility effects and also contained no SNR confound. In some cases, there was little or no SNR effect because performance was nearly equal between the $\mathrm{NH}$ and $\mathrm{HI}$ listeners in a stationary-noise background as a result of the addition of background noise or addition of gain. In these cases, the HI listeners showed very little reduction in FMB $(0-2 \mathrm{~dB})$ relative to the NH listeners (Bacon et al., 1998; Phatak and Grant, 2012, 2014), except with a high-rate modulated-noise masker (Phatak and Grant, 2012). Similarly, Léger et al. (2012a) employed speech stimuli that were bandlimited to a spectral region where $\mathrm{HI}$ listeners had normal or near-normal audiometric thresholds, and found no reduction in FMB relative to $\mathrm{NH}$ listeners.

Two previous studies explicitly controlled both audibility and SNR effects and came to very different conclusions. Jin and Nelson (2006) applied individualized gain for the HI listeners, and showed a $20-40$ percentage-point reduction in the FMB for HI relative to NH listeners at a given fixed SNR. Desloge et al. (2010) applied noise masking and expansion to the signals presented to the $\mathrm{NH}$ listeners, and found generally similar FMB for the NH and HI listeners.

To understand why these studies that apparently controlled both SNR and audibility effects came to different conclusions regarding the effects of hearing loss on the FMB, it is instructive to compare the specific methods that were used to control audibility effects. For two of the cases where the HI listeners exhibited similar FMB to the NH listeners, masking noise was used to simulate reduced audibility in the NH listeners (Bacon et al., 1998; Desloge et al., 2010). In another case, equal audibility was ensured by limiting the bandwidth of the stimuli (Léger et al., 2012a). In contrast, Jin and Nelson (2006) applied individualized gain to improve audibility for the HI listeners, and still found a reduced FMB. Phatak and Grant $(2012,2014)$ also took the approach of providing individualized gain to the HI listeners to restore audibility. They found a similar amount of FMB for the NH and $\mathrm{HI}$ listeners for cases with low-rate modulations, but only in the cases of vowel perception, where an analysis of the speech spectrogram showed that the individualized gain restored audibility. For consonants, HI listeners still showed reduced FMB, but the application of gain was not successful in restoring audibility for these relatively lowintensity speech cues. In summary, HI listeners have generally shown a reduced FMB relative to $\mathrm{NH}$ listeners only in those cases that attempted to use gain to restore audibility to the HI listeners. This suggests that the FMB was reduced for 
the HI listeners because audibility was not completely restored, especially for the lower portion of the speech dynamic range that are uncovered during gaps in the masking noise.

\section{Effect of masker type}

The current study found that even when audibility and SNR effects were controlled, the HI listeners still showed reduced FMB, but only for the $32-\mathrm{Hz}$ SAM masker. For a low-rate modulated masker, these results suggest that the main factor limiting FMB for HI listeners is a lack of audibility of speech information falling below absolute threshold. This interpretation was supported by two observations. First, once audibility was equalized for the NH and HI listeners, there was no difference in FMB between the two listener groups for the 4-HZ SAM-noise masker [Fig. 5(B)], similar to what was observed for low-rate $(2-10 \mathrm{~Hz})$ modulatednoise maskers in previous studies (Desloge et al., 2010; Phatak and Grant, 2012, 2014). Second, the magnitude of the FMB for the 4-Hz SAM-noise masker was strongly related to the HI audiometric threshold [Fig. 6(B)]. The HI thresholds determined the criterion level for the intensity-filtering for each HI listener and their matched NH counterpart. The fact that the FMB strongly depended on this criterion suggests that intersubject variability in the FMB for a low-rate masker directly reflects audibility.

For a high-rate $(32-\mathrm{Hz})$ SAM-noise modulated masker, the FMB for HI listeners was reduced by about $4.5 \mathrm{~dB}$, similar to what was observed by Phatak and Grant (2012). The fact that $\mathrm{HI}$ listeners experience a reduced FMB for highrate $(32 \mathrm{~Hz})$ but not for low-rate $(4 \mathrm{~Hz})$ modulated-noise maskers suggests that the deficit is specifically attributable to temporal resolution, and that $\mathrm{HI}$ listeners do not have a general problem "listening in the dips" of a fluctuating masker that cannot be explained by audibility effect. The fact that the FMB was unrelated to audiometric threshold [Fig. 6(C)] further supports the idea that audibility was not the main cause of the reduced FMB for $\mathrm{HI}$ listeners for the $32-\mathrm{Hz}$ SAM masker. In the case of an interfering talker, there was no strong evidence that the FMB was reduced for HI listeners, but the presence of a non-significant trend [Fig. 5(A)] suggests that we cannot rule out the possibility that the FMB might have been reduced for the HI listeners.

\section{Temporal processing}

The effect of modulation rate on the FMB observed in the current study suggests that the HI listeners experienced a deficit in temporal processing that prohibited the extraction of target speech information from the brief dips in the $32-\mathrm{Hz}$ SAM-noise masker. The most likely explanation for this effect is that the hearing loss caused a reduction in temporal resolution that manifested in reduced FMB only for the high-rate SAM noise. For the other fluctuating maskers with slower fluctuations, the degree of temporal-resolution impairment would have been small relative to the period of the modulations, thereby having much less of an effect. Although HI listeners do not generally demonstrate any deficit in the detection or discrimination of temporal modulation
(Bacon and Gleitman, 1992; Füllgrabe et al., 2003), cochlear compression associated with hearing loss can lead to a decrease in temporal resolution in the form of increased forward masking (e.g., Nelson et al., 2001). Thus, the reduced FMB for HI listeners for a high-rate fluctuating masker likely reflects excess forward masking of the speech content occurring during very short glimpses.

Like the current study, Phatak and Grant (2012) also observed an effect of hearing loss on the FMB for high-rate $(32 \mathrm{~Hz})$ but not for lower rate $(2-12 \mathrm{~Hz})$ modulated maskers. However, it was not clear in their study whether this deficit was attributable to aging or hearing loss. Although there was a non-significant trend for their agematched older NH listeners to show less FMB than their older HI listeners, the only significant effect of hearing loss on the FMB was observed when comparing the younger $\mathrm{NH}$ and older HI listener groups. In fact, there is evidence from the literature that aging per se can reduce the FMB for high-rate modulated maskers. Dubno et al. (2003) identified an age-related decline in temporal processing for elderly listeners, with the identification of speech in the presence of a high-rate $(25-50 \mathrm{~Hz})$ modulated masking noise related to a deficit in forward masking thresholds for tone detection. George et al. (2006) reported that the FMB for highrate modulated maskers was correlated to both age and temporal resolution. In the current study, it is unlikely that an age-related decline in temporal resolution was responsible for the observed reduction in FMB for a high-rate $(32-\mathrm{Hz})$ SAM-noise masker, since the $\mathrm{NH}$ and $\mathrm{HI}$ listeners were age matched. Thus, these results suggest that the observed temporal-processing deficit is directly related to the hearing loss.

One methodological detail that could have influenced the experimental results for the $32-\mathrm{Hz}$ masker condition was that the 17-ms duration of each gap in the modulated masker was comparable to the 20-ms time-segmentation employed by the intensity-filtering analysis. This raises the possibility that the processing could have influenced the audibility of the speech during the masker gaps, such that audibility differences might still have contributed to the reduced FMB observed for HI listeners. However, the likelihood of this occurring was mitigated by the fact that the speech and masking noise were each intensity-filtered separately before being added together. This means that just as for the lowrate maskers, only speech segments falling above the HI absolute threshold would have been presented. Furthermore, the effective result of the processing applied to the masker was to either leave the masker modulations intact, or to delete the noise entirely in a given frequency bin. Because the modulation period was on the order of the resolution of the intensity filtering, any noise falling above the threshold criteria was passed through unprocessed, leaving the gaps in place. Any noise falling below the criterion was removed completely by the algorithm, but in these cases, any lowlevel speech information that would have appeared during the gap was also removed. These factors meant that, suprathreshold deficits aside, any speech segment audible to the $\mathrm{NH}$ listener during dips in the masker level would also have been audible to the HI listener. 


\section{E. The perceptual separation of concurrent talkers}

There was only a non-significant trend in the data suggesting that $\mathrm{HI}$ listeners might have experienced a reduced FMB in the case of interfering speech. If there was an effect of hearing loss on the FMB, the magnitude of this effect appeared to be small, on the order of about $2 \mathrm{~dB}$ [Fig. 5(A)]. This result contrasts with other studies in the literature that have identified a clear reduction in FMB for HI listeners in the case of interfering speech (Bernstein and Grant, 2009; Christiansen and Dau, 2012; Festen and Plomp, 1990; Peters et al., 1998; Strelcyk and Dau, 2009). The major difference between these studies and the current study is that the current study controlled both audibility and SNR, while these previous studies did not, suggesting that once these factors are controlled, hearing loss has very little effect on the ability to understand speech in the presence of an opposite-gender interfering talker.

To the extent that there was any reduction in FMB for the HI listeners in the case of an interfering talker, this deficit is unlikely to be attributable to reduced temporal resolution. A speech masker is dominated by low-rate modulations (2-8 Hz; Steeneken and Houtgast, 1980), and there was no FMB reduction observed for $\mathrm{HI}$ listeners for a low-rate modulated-noise masker. (Although, to be certain that this was an effect specific to speech-on-speech masking, it would be important to test a condition with speech-envelope modulated noise, where the masker modulations are designed to be more similar to those of a speech masker; e.g., Grant and Walden, 2013). Instead, hearing impairment might distort some of the cues on which listeners rely to perceptually separate concurrent voices in a mixture. For example, NH listeners have been shown to benefit more than HI listeners from the addition of temporal fine-structure information to the signal in a speech-on-speech masking task (Hopkins and Moore, 2009; Lunner et al., 2012). One interpretation of this result is that NH listeners are able to make use of the TFS information that encodes information regarding voice pitch to perceptually separate concurrent voices, but that HI listeners have a reduced ability to do so. In the current study, the interfering speech was produced by a female talker, whereas the target speech was produced by male talkers. In contrast, the perceptual separation of concurrent talkers is much more difficult when the target and interfering speech is produced by talkers of the same gender (e.g., Brungart et al., 2001). This leaves open the possibility that the FMB might be significantly reduced by hearing loss in cases of same-gender interfering talkers, where distorted perceptual-separation cues could have an even larger impact.

\section{F. The impact of hearing loss on speech perception in stationary noise}

While the main goal of the current study was to examine the effect of hearing loss on speech perception in the presence of fluctuating maskers, it is also instructive to examine performance in the stationary-noise masker condition to examine the extent to which HI performance can be attributed to audibility or supra-threshold-processing factors. Hearing loss did not significantly affect the set size required to achieve a given level of performance in stationary noise. This lack of effect of hearing loss on speech-reception performance in noise is surprising given the vast literature suggesting that $\mathrm{HI}$ listeners experience considerable variability in understanding speech in noise that cannot be accounted for by differences in audiometric threshold (Amos and Humes, 2007; Bernstein et al., 2013, 2016; Davies-Venn et al., 2015; Léger et al., 2012a,b). On the other hand, this result is consistent with several previous studies showing that once audibility was equalized for the NH and HI listeners, the HI listeners experienced little or no deficit for speech understanding with a stationary-noise masker (Bacon et al., 1998; Jin and Nelson, 2006; Phatak and Grant, 2012, 2014).

One possible interpretation of these conflicting sets of results is that on average, hearing impairment results in very little deficit for speech understanding in noise that cannot be accounted for by audibility, but there is nevertheless considerable inter-subject variability not directly related to the hearing loss that reflects other factors such as individual differences in cognitive function (Humes, 2007). Another interpretation is that $\mathrm{HI}$ listeners might experience less modulation interference from inherent fluctuations in the stationary-noise masker (Stone et al., 2012; Stone and Moore, 2014) due to broader peripheral tuning, as has been demonstrated for cochlear-implant listeners (Oxenham and Kreft, 2014). Reduced modulation interference could offset other putative supra-threshold deficits associated with hearing loss.

\section{G. Implications for hearing-aid processing}

Our results indicated that HI listeners experience suprathreshold deficits in dip listening mainly when speech is presented in a background dominated by high-rate modulations - in other words, when the dips are brief (on the order of $17 \mathrm{~ms}$ at $32 \mathrm{~Hz}$ ). For maskers dominated by slower modulations, HI listeners experience little difficulty in extracting speech information from dips in the masker level, provided that target speech fragments unmasked by dips in the masker level are audible above the absolute threshold. To the extent that audibility limitations are the predominant factor limiting the FMB for HI listeners, at least for low-rate modulated maskers, the current results suggest that difficulties extracting speech information from fluctuating backgrounds might be overcome, at least to some extent, with hearing-aid signal processing. Although Phatak and Grant (2012) found that linear prescriptive gain cannot restore audibility for low-level consonant information, several researchers have investigated how nonlinear compression schemes can amplify low-level target speech occurring in the dips of a fluctuating masker to improve performance for HI listeners. For example, Desloge et al. (2017) and D'Aquila et al. (2017) tested an algorithm that selectively amplifies short, low-level portions of a combined speech and noise signal, and found that the algorithm improved performance for maskers containing regular temporal fluctuations. A standard hearing-aid fast-compression algorithm might also improve speech understanding in a fluctuating background (Lunner and Sundewall-Thorén, 2007), especially at 
low SNRs (Naylor and Johannesson, 2009), although Stone and Moore (2008) cautioned that such an approach could introduce distortion in a speech-on-speech masking situation.

\section{SUMMARY AND CONCLUSIONS}

This study examined the effect of hearing loss on speech perception in the presence of a stationary or fluctuating masker. The size of the response set was adjusted to equalize speech-reception performance in stationary noise, while an intensity-filtering algorithm was used to equalize signal audibility while presenting identical signals to age-matched pairs of $\mathrm{NH}$ and $\mathrm{HI}$ listeners. Relative to the $\mathrm{NH}$ listeners, the $\mathrm{HI}$ listeners showed a clear $4.5-\mathrm{dB}$ reduction in the magnitude of the FMB for a 32-Hz SAM-noise masker, no reduction in FMB for a 4-Hz SAM-noise masker, and only a nonsignificant trend toward a $2-\mathrm{dB}$ reduction in FMB for an interfering talker of opposite gender from the target speech. Overall, these results suggest that once audibility and SNR effects have been controlled, HI listeners do not show a general deficit in the ability to take advantage of momentary dips in the level of a fluctuating masker. Rather, hearing loss causes a specific temporal-processing deficit that reduces dip-listening ability for high-rate fluctuating maskers and might also reduce the salience of cues needed perceptually separate concurrent talkers. In conjunction with the important role of audibility in facilitating the FMB, the lack of a general dip-listening deficit for HI listeners bodes well for the potential success of signal-processing solutions that aim to amplify low-level speech cues occurring during dips in the level of a fluctuating masker.

\section{ACKNOWLEDGMENTS}

We thank Ken Grant, Sandeep Phatak, two anonymous reviewers, and the Associate Editor, Virginia Best, for helpful comments on a previous version of this manuscript. We also thank Ken Grant and Doug Brungart for helpful discussions in planning the study. Research reported in this publication was supported by the National Institute On Deafness and Other Communication Disorders of the National Institutes of Health under Award No. R03 DC010264 (J.G.W.B.). The content is solely the responsibility of the authors and does not necessarily represent the official views of the National Institutes of Health. The views expressed in this article are those of the authors and do not reflect the official policy of the Department of Army/Navy/Air Force, Department of Defense, or U.S. Government. The identification of specific products or scientific instrumentation does not constitute endorsement or implied endorsement on the part of the author, DoD, or any component agency.

Amos, N. E., and Humes, L. E. (2007). "Contribution of high frequencies to speech recognition in quiet and noise in listeners with varying degrees of high-frequency sensorineural hearing loss," J. Speech Lang. Hear. Res. 50, 819-835.

Bacon, S. P., and Gleitman, R. M. (1992). "Modulation detection in subjects with relatively flat hearing losses,” J. Speech Hear. Res. 35, 642-653.
Bacon, S. P., Opie, J. M., and Montoya, D. Y. (1998). "The effects of hearing loss and noise masking on the masking release for speech in temporally complex backgrounds," J. Speech Lang. Hear. Res. 41, 549-563.

Bernstein, J. G. W. (2010). "Why do hearing-impaired listeners fail to benefit from masker fluctuations?," in The Neurophysiological Bases of Auditory Perception, edited by E. A. Lopez-Poveda, R. Meddis, and A. R. Palmer (Springer, New York), pp. 609-619.

Bernstein, J. G. W. (2012). "Controlling signal-to-noise ratio effects in the measurement of speech intelligibility in fluctuating maskers," in Speech Perception and Auditory Disorders, edited by T. Dau, C. Dalsgaard, M. L. Jepsen, and T. Poulsen (Danavox Jubilee Foundation, Lautrupbjerg, Denmark), pp. 33-44.

Bernstein, J. G. W., and Brungart, D. S. (2011). "Effects of spectral smearing and temporal fine-structure distortion on the fluctuating-masker benefit for speech at a fixed signal-to-noise ratio," J. Acoust. Soc. Am. 130, 473-488.

Bernstein, J. G. W., Danielsson, H., Hällgren, M., Stenfelt, S., Rönnberg, J., and Lunner, T. (2016). "Spectrotemporal modulation sensitivity as a predictor of speech-reception performance in noise with hearing aids," Trends Hear. 20, 2331216516670387.

Bernstein, J. G. W., and Grant, K. W. (2009). "Auditory and auditory-visual intelligibility of speech in fluctuating maskers for normal-hearing and hearing-impaired listeners," J. Acoust. Soc. Am. 125, 3358-3372.

Bernstein, J. G. W., Mehraei, G., Shamma, S., Gallun, F. J., Theodoroff, S. M., and Leek, M. R. (2013). "Spectrotemporal modulation sensitivity as a predictor of speech intelligibility for hearing-impaired listeners," J. Am. Acad. Audiol. 24, 293-306.

Bernstein, J. G. W., Summers, V., Iyer, N., and Brungart, D. S. (2012). "Setsize procedures for controlling variations in speech-reception performance with a fluctuating masker," J. Acoust. Soc. Am. 132, 2676-2689.

Brown, G. J., and Wang, D. (2005). "Separation of speech by computational auditory scene analysis," in Speech Enhancement, edited by J. Benesty, S. Makino, and J. Chen (Springer, New York), pp. 371-402.

Brungart, D. S., and Iyer, N. (2012). "Better-ear glimpsing efficiency with symmetrically-placed interfering talkers," J. Acoust. Soc. Am. 132, 2545-2556.

Brungart, D. S., Simpson, B. D., Ericson, M. A., and Scott, K. R. (2001). "Informational and energetic masking effects in the perception of multiple simultaneous talkers," J. Acoust. Soc. Am. 110, 2527-2538.

Christiansen, C., and Dau, T. (2012). "Relationship between masking release in fluctuating maskers and speech reception thresholds in stationary noise," J. Acoust. Soc. Am. 132, 1655-1666.

Cooke, M. (2006). "A glimpsing model of speech perception in noise," J. Acoust. Soc. Am. 119, 1562-1573.

D’Aquila, L. A., Desloge, J. G., Reed, C. M., and Braida, L. D. (2017). "Effect of energy equalization on the intelligibility of speech in fluctuating background interference for listeners with hearing impairment," Trends Hear. 21, 2331216517710354.

Davies-Venn, E., Nelson, P., and Souza, P. (2015). "Comparing auditory filter bandwidths, spectral ripple modulation detection, spectral ripple discrimination, and speech recognition: Normal and impaired hearing," J. Acoust. Soc. Am. 138, 492-503.

Desloge, J. G., Reed, C. M., Braida, L. D., Perez, Z. D., and D’Aquila, L. A. (2017). "Masking release for hearing-impaired listeners: The effect of increased audibility through reduction of amplitude variability," J. Acoust. Soc. Am. 141, 4452-4465.

Desloge, J. G., Reed, C. M., Braida, L. D., Perez, Z. D., and Delhorne, L. A. (2010). "Speech reception by listeners with real and simulated hearing impairment: Effects of continuous and interrupted noise," J. Acoust. Soc. Am. 128, 342-359.

Drullman, R. (1995). "Speech intelligibility in noise: Relative contribution of speech elements above and below the noise level," J. Acoust. Soc. Am. 98, 1796-1798.

Dubno, J. R., Horwitz, A. R., and Ahlstrom, J. B. (2003). "Recovery from prior stimulation: Masking of speech by interrupted noise for younger and older adults with normal hearing," J. Acoust. Soc. Am. 113, 2084-2094.

Eisenberg, L. S., Dirks, D. D., and Bell, T. S. (1995). "Speech recognition in amplitude-modulated noise of listeners with normal and listeners with impaired hearing," J. Speech Lang. Hear. Res. 38, 222-233.

Festen, J. M. (1993). "Contributions of comodulation masking release and temporal resolution to the speech-reception threshold masked by an interfering voice," J. Acoust. Soc. Am. 94, 1295-1300.

Festen, J. M., and Plomp, R. (1990). "Effects of fluctuating noise and interfering speech on the speech-repception threshold for impaired and normal hearing," J. Acoust. Soc. Am. 88, 1725-1736. 
Fousek, P., Svojanovský, P., Grézl, F., and Hermansky, H. (2004). "New nonsense syllables database-Analyses and preliminary ASR experiments," in Proceedings of the 8th International Conference on Spoken Language Processes (ICSLP), Jeju, South Korea.

Freyman, R. L., Griffin, A. M., and Oxenham, A. J. (2012). "Intelligibility of whispered speech in stationary and modulated noise maskers," J. Acoust. Soc. Am. 132, 2514-2523.

Füllgrabe, C., Meyer, B., and Lorenzi, C. (2003). "Effect of cochlear damage on the detection of complex temporal envelopes," Hear. Res. 178, 35-43.

George, E. L. J., Festen, J. M., and Houtgast, T. (2006). "Factors affecting masking release for speech in modulated noise for normal-hearing and hearing-impaired listeners," J. Acoust. Soc. Am. 120, 2295-2311.

Grant, K. W., and Walden, T. C. (2013). "Understanding excessive SNR loss in hearing-impaired listeners," J. Am. Acad. Audiol. 24, 258-273; quiz 337-8.

Gustafsson, H. ^., and Arlinger, S. (1994). "Masking of speech by amplitude-modulated noise," J. Acoust. Soc. Am. 95, 518-529.

Hall, J. W., Buss, E., Grose, J. H., and Roush, P. A. (2012). "Effects of age and hearing impairment on the ability to benefit from temporal and spectral modulation," Ear Hear. 33, 340-348.

Hopkins, K., and Moore, B. C. J. (2009). "The contribution of temporal fine structure to the intelligibility of speech in steady and modulated noise," J. Acoust. Soc. Am. 125, 442-446.

Humes, L. E. (2007). "The contributions of audibility and cognitive factors to the benefit provided by amplified speech to older adults," J. Am. Acad. Audiol. 18, 590-603.

Jin, S.-H., and Nelson, P. B. (2006). "Speech perception in gated noise: The effects of temporal resolution," J. Acoust. Soc. Am. 119, 3097-3108.

Léger, A. C., Moore, B. C. J., and Lorenzi, C. (2012a). "Temporal and spectral masking release in low- and mid-frequency regions for normalhearing and hearing-impaired listeners," J. Acoust. Soc. Am. 131, 1502-1514.

Léger, A. C., Moore, B. C. J., and Lorenzi, C. (2012b). "Abnormal speech processing in frequency regions where absolute thresholds are normal for listeners with high-frequency hearing loss," Hear. Res. 294, 95-103.

Levitt, H. (1971). "Transformed up-down methods in psychoacoustics," J. Acoust. Soc. Am. 49, 467-477.

Lorenzi, C., Husson, M., Ardoint, M., and Debruille, X. (2006). "Speech masking release in listeners with flat hearing loss: Effects of masker fluctuation rate on identification scores and phonetic feature reception,” Int. J. Audiol. 45, 487-495.

Lunner, T., Hietkamp, R. K., Andersen, M. R., Hopkins, K., and Moore, B. C. J. (2012). "Effect of speech material on the benefit of temporal fine structure information in speech for young normal-hearing and older hearing-impaired participants," Ear Hear. 33, 377-388.

Lunner, T., and Sundewall-Thorén, E. (2007). "Interactions between cognition, compression, and listening conditions: Effects on speech-in-noise performance in a two-channel hearing aid," J. Am. Acad. Audiol. 18, 604-617.

Miller, G. A., and Licklider, J. C. R. (1950). "The intelligibility of interrupted speech,” J. Acoust. Soc. Am. 22, 167-173.
Moore, B. C. J., and Glasberg, B. R. (1998). "Use of a loudness model for hearing-aid fitting. I. Linear hearing aids,” Br. J. Audiol. 32, 317-335.

Naylor, G., and Johannesson, R. B. (2009). "Long-term signal-to-noise ratio at the input and output of amplitude-compression systems," J. Am. Acad. Audiol. 20, 161-171.

Nelson, D. A., Schroder, A. C., and Wojtczak, M. (2001). "A new procedure for measuring peripheral compression in normal-hearing and hearingimpaired listeners," J. Acoust. Soc. Am. 110, 2045-2064.

Oxenham, A. J., and Kreft, H. A. (2014). "Speech perception in tones and noise via cochlear implants reveals influence of spectral resolution on temporal processing," Trends Hear. 18, 2331216514553783.

Oxenham, A. J., and Simonson, A. M. (2009). "Masking release for lowand high-pass-filtered speech in the presence of noise and single-talker interference," J. Acoust. Soc. Am. 125, 457-468.

Peters, R. W., Moore, B. C. J., and Baer, T. (1998). "Speech reception thresholds in noise with and without spectral and temporal dips for hearing-impaired and normally hearing people," J. Acoust. Soc. Am. 103, 577-587.

Phatak, S. A., and Grant, K. W. (2012). "Phoneme recognition in modulated maskers by normal-hearing and aided hearing-impaired listeners," J. Acoust. Soc. Am. 132, 1646-1654.

Phatak, S. A., and Grant, K. W. (2014). "Phoneme recognition in vocoded maskers by normal-hearing and aided hearing-impaired listeners," J. Acoust. Soc. Am. 136, 859-866.

Steeneken, H. J., and Houtgast, T. (1980). "A physical method for measuring speech-transmission quality," J. Acoust. Soc. Am. 67, 318-326.

Stone, M. A., Füllgrabe, C., and Moore, B. C. J. (2012). "Notionally steady background noise acts primarily as a modulation masker of speech," J. Acoust. Soc. Am. 132, 317-326.

Stone, M. A., and Moore, B. C. J. (2008). "Effects of spectro-temporal modulation changes produced by multi-channel compression on intelligibility in a competing-speech task," J. Acoust. Soc. Am. 123, 1063-1076.

Stone, M. A., and Moore, B. C. J. (2014). "On the near non-existence of 'pure' energetic masking release for speech," J. Acoust. Soc. Am. 135, 1967-1977.

Strelcyk, O., and Dau, T. (2009). "Relations between frequency selectivity, temporal fine-structure processing, and speech reception in impaired hearing," J. Acoust. Soc. Am. 125, 3328-3345.

ter Keurs, M., Festen, J. M., and Plomp, R. (1993). "Effect of spectral envelope smearing on speech reception II," J. Acoust. Soc. Am. 93, $1547-1552$.

Versfeld, N. J., and Dreschler, W. A. (2002). "The relationship between the intelligibility of time-compressed speech and speech in noise in young and elderly listeners," J. Acoust. Soc. Am. 111, 401-408.

Zeng, F.-G., Grant, G., Niparko, J., Galvin, J., Shannon, R., Opie, J., and Segel, P. (2002). "Speech dynamic range and its effect on cochlear implant performance," J. Acoust. Soc. Am. 111, 377-386.

Zwislocki, J. J., and Relkin, E. M. (2001). "On a psychophysical transformed-rule up and down method converging on a 75\% level of correct responses,” Proc. Natl. Acad. Sci. U.S.A. 98, 4811-4814. 